\title{
A Neural Model of Hand Grip formation during Reach to Grasp
}

\author{
Javier Molina Vilaplana, Jorge Feliú Batlle, Juan López Coronado \\ Department of Automatics and Systems Engineering. \\ Technical University of Cartagena \\ $\mathrm{C} /$ Dr Fleming S/N 30202 \\ Cartagena. Murcia. Spain \\ Javi.Molina@upct.es, Jorge.Feliu@upct.es, JL.Coronado@upct.es.
}

\begin{abstract}
In this paper, we investigate the spatio temporal dynamics of hand pre-shaping during prehension through a biologically plausible neural network model. It is proposed that the hand grip formation in prehension can be understood in terms of basic motor programs that can be rescaled both spatially and temporally to accommodate different task demands. The model assumes a timing coordinative role to propioceptive reafferent information generated by the reaching component of the movement, avoiding the need of a pre-organized functional temporal structure for the timing of prehension as some previous models have proposed. Predictions of the model in both Normal and Altered initial hand aperture conditions match key kinematic features present in human data. The differences between the proposed model and previous models predictions are used to try to identify the major principles underlying prehensile behavior.
\end{abstract}

Keywords: Reach to Grasp, Neural Models, Human Data, Neurorobotics.

\section{Introduction}

To grasp an object, the central nervous system (CNS) must program two simultaneous motor actions: hand transport to the location of the object to be grasped and hand pre-shaping to enclose object [1], [2]. The preshaping movement of the fingers during prehension follows a stereotyped kinematic pattern: from a closed initial position (thumb and index fingers in slight contact), the thumb and fingers begin to separate until maximum hand aperture is reached. As the wrist approaches to the object location, the fingers begin to close, adapting to object's size and shape. There is a parallel evolution of reaching and hand pre-shaping processes, both of them initiating and finishing nearly simultaneously. The time of maximum grip aperture is well correlated with the time of maximum deceleration of the transport component [1]. According to Jeannerod [3] and Arbib [4], the transport and hand pre-shaping components evolve independently trough two segregated visuomotor channels, coordinated trough a central pre-organized functional temporal structure. This mechanism should ensure the temporal alignment of key moments in the evolution of the two components: in this way, Jeannerod [3] suggested that this central timing mechanism operates such that peak hand aperture is reached at the moment of peak deceleration of the reaching component

Several studies have demonstrated a peculiar effect of initial hand aperture on the grip formation in reach to grasp movements. Saling et al [5] and Timman et al [6] reported that for precision grip movements starting with an initial finger aperture, the hand aperture closed and then reopened before maximum grip aperture was achieved. This was remarkable as it was expected a monotonic closing of the hand towards the object. Saling et al [5] hypothesized that if grip reorganization is functional, it should be related to object size. They reported that for the Altered condition, the time and amplitude of the first change in the initial closing finger aperture profile scales with object size. Also evident was that the relative timing between late manipulation and transport components remained constant across conditions. Wallace et al [7] also reported that regardless of different initial grip postures, very small changes were observed in the temporal occurrence of maximum grip aperture and the onset of final grip closure relative to the overall duration of movement. Recently, predictions of two different models [8], [9] for prehension have been compared with the findings mentioned above [10]. These two models can account for many aspects of human grasping when the movement starts with the digits in contact but both models predict an initial hand opening in the Altered initial hand aperture that is not present in the human data.

In this paper a neural model of hand pre-shaping formation during reach to grasp is proposed. We

\footnotetext{
* 0-7803-8566-7/04/\$20.00 C 2004 IEEE
} 
investigate the spatio-temporal dynamics of hand preshaping (or hand gestures) for anticipatory finger movements in terms of possible dynamic neural representations of motor programs for prehension. Vector Integration To Endpoint (VITE) [11] dynamics is used to model the two segregated visuomotor channels related with grip formation and wrist transport towards the object to be grasped. It is proposed that the movement preshaping of hand and fingers in prehension can be understood in terms of basic motor programs that can be rescaled both temporally and spatially to accommodate different task demands [12]. Based on the work of Santello et al [13], it is hypothesized that the neural representation for these motor programs evolves gradually over time. The model also introduce a timing coordinative role to propioceptive reafferent information related with the reaching movement, avoiding the need of a preorganized functional temporal structure for the reach to grasp temporal coordination [3], [4]. In that sense, in the present model the hand pre-shaping dynamics during prehension emerges from the combined action of the feedforward motor program execution and the temporal coordinative role of some propioceptive reafferent information related with the transport phase of the movement. Predictions of the model in both Normal and Altered initial hand aperture conditions match key kinematic features of human data. The differences between the presented model and previous models predictions [10] are used to try to determine the major neural principles underlying prehensile behaviour.

\section{Computational Model}

The pre-shaping of the manipulation component has been simulated using the VITE model of Bullock and Grossberg [11]. The hand aperture is modelled as one degree of freedom (DOF) system. This corresponds to modelling the distance (aperture) between thumb and index finger directly. Computationally speaking, the VITE system gradually integrates the difference between the desired target finger aperture $(\mathrm{T})$ and the actual finger aperture (P) to obtain a difference vector (V). The difference vector codes information about the amplitude and direction of the desired movement, and its is modulated by a time-varying $\mathrm{GO}$ signal to produce a desired finger aperture velocity (V.G(t), [14], [15]). The outflow command V.G(t) is integrated to update the finger aperture (P) as shown in equations (1) - (3).

$$
\begin{aligned}
& \frac{d V}{d t}=30(-V+T-P) \\
& \frac{d P}{d t}=G(t) \cdot V \\
& G(t)=g \frac{t^{2}}{\left(0.25+t^{2}\right)}
\end{aligned}
$$

We have modified the VITE model to account for the apparent gradual specification of target amplitude [16] (Fig 1). Motor and premotor cortical cells are modulated by distance and target along at least one direction during pre-movement and movement periods. Furthermore, the correlation of cell discharge with movement distance increased towards the end of movement, supporting the idea of a gradual specification of movement extent. The motor program for the grasp component was modelled as a biphasic motor program consisting of two successive subprograms (G1, G2), where $\mathrm{G1}$ is related to the maximum grip aperture and G2 is equal to the object size. It is hypothesized that the intrinsic properties of the object such as object size may be computed in advance of the onset, whereas the acquisition of the target aperture may evolve even after aperture onset. The model assumes that the target aperture is not fully programmed in $T$ before movement initiation; rather, it is postulated that $T$ in grasp channel, sequentially and gradually specify, in a first phase the desired maximum grip aperture (G1), and in a second phase, the hand configuration corresponding to the object size (G2). The proposed modification in $\mathrm{T}$ dynamics of grasp channel is described by equation (4),

$$
\frac{d T}{d t}=\beta\left(-T+G_{i}\right)
$$

where $\beta$ is a rate of integration.

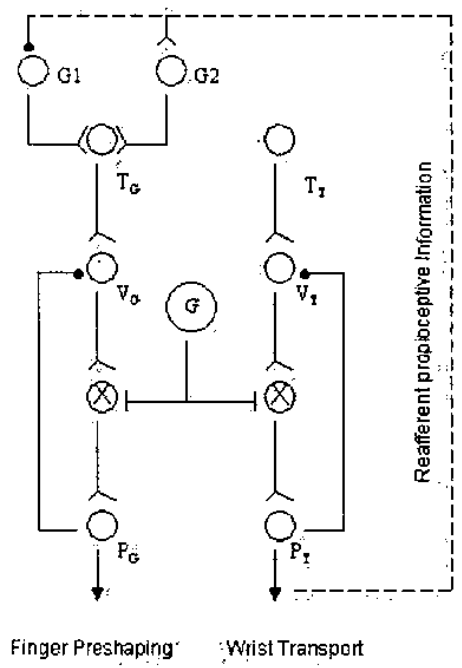

Figure 1. Neural network model for hand grip formation. Transport (T) and grasp (G) phases of the movement are modeled using VITE model. Inhibitory connections are

represented as lines finishing in a circle. Excitatory connections are represented as an open arrow.

In the case of the transport component, classical VITE dynamics has been used to simulate the wrist position evolution through movement. A single and 
constant over time input, fully specified the target distance. Simoneau et al [17], have suggested that during a unimanual reach to grasp task, propioceptive reafferents are used to coordinate transport and grasp motor program execution by the two related neural channels. These authors concluded that reafferent propioceptive information from the reaching movement is used by the CNS to coordinate the reaching and grasping movements. These results do not agree with the hypothesis that coordination between reaching and grasping pertains to a pre-organized functional temporal structure for unimanual reach to grasp movement [3], [4]. In the model, we have adopted the hypothesis of [17] and, detection of a minimum in transport acceleration triggered the read-in of G2 (e.g., hand enclosing begins at the instant when peak deceleration in transport channel (tpdec) is detected). In principle, proprioceptive information could be used by the CNS to derive tpdec or a related measurement [18]. No other interactions between transport and manipulation components were assumed as transport component in previous studies were not influenced by manipulation component ([5], [6], [7]).

\section{Model Simulations}

To study the pre-shaping movement of fingers under Normal and Altered initial grip aperture in reach to grasp to small and large objects, we have simulated single prehension movements of two different size objects, a small object $(20 \mathrm{~mm})$ and a large object $(70 \mathrm{~mm})$. In the Normal condition the initial finger aperture was set to zero (in grasp channel, $P=0 \mathrm{~mm}$ before movement onset). In the Altered condition, the initial finger aperture was set to $P=110 \mathrm{~mm}$. The biphasic motor program in the grasp channel $(\mathrm{G} 1, \mathrm{G} 2)$ was designed taking into account the fact that the amplitude of maximum grip aperture covaries linearly with object size. Grasp motor program for small object was stated as ( $G 1=60 \mathrm{~mm}, \mathrm{G} 2=20 \mathrm{~mm}$ ). Grasp motor program for the large object was stated as $(\mathrm{G} 1=110$ $\mathrm{mm}, \mathrm{G} 2=70 \mathrm{~mm}$ ). For transport component of the movement, motor program was described by $\mathrm{T}$ and set to $300 \mathrm{~mm}$. Initial wrist position was fixed at $\mathrm{P}=0 \mathrm{~mm}$. In all simulations $g=15$ and $\beta=10$. Equations (1) - (4) were integrated during 1200 simulation steps by a Runge-Kutta integration method of fourth order with fixed step set to $h$ $=0.001$. Each simulation step corresponds to 1 millisecond ( $\mathrm{ms}$ ) in real time.

\section{Discussion}

\subsection{Model Performance}

The model is able to reproduce the grip kinematics for all the initial conditions and object sizes. Although the Figure. 2. Grip Aperture (A) and grip aperture velocity (B) profiles for a small (up) and a large (down) object between hand pre-closing in the Altered condition could suggest a functional reorganization of the grip kinematics [5], the present simulations suggest that instead the altered grip kinematics may result from a target aperture that has not been fully specified at movement onset ([18], [19]). In the model, as the not-fully specified target aperture ( $T$ in grasp channel) is smaller than the non-zero initial aperture in the Altered condition ( $P=110 \mathrm{~mm}$ ), the finger aperture ( $P$ in grasp channel) will tend to close due to equation (1). This closing of grip aperture finish when the target acquisition neuron $\mathrm{T}$ that is gradually evolving (equation (4)) to maximum grip aperture specified by $\mathrm{G1}$, matches the current aperture. At this time, due to V dynamics, the hand aperture begins to increase until it reaches a maximum grip aperture in preparation for the hand enclosing. From the point of view of the model, there is no existence of a functional reorganization of the grip kinematics in the Altered condition. The grip kinematics emerging from the Altered initial condition is consequence of the neural dynamics involved in trajectory formation modelled by VITE and the assumption that 1) object's intrinsic properties are computed (and coded in motor program $(\mathrm{G} 1, \mathrm{G} 2)$ in advance of the movement onset and 2) the idea of a gradual specification of the motor program in target acquisition neuron in grasp channel $(\mathrm{T})$. In this framework, the second modulation in grip aperture can not be considered as the correction of a pre-programmed movement as was suggested in [5]. In terms of velocity profiles, the model is able to reproduce the bi-phasic and tri-phasic grip velocity patterns during hand pre-shaping in Normal and Altered initial finger conditions respectively (Fig $2 \mathrm{~B}$ ).

In simulations and due to our neural model architecture, transport component were not influenced by manipulation component as previous studies by [5], [6] and [7] had noted. In that sense, in all simulated movements, time to peak deceleration (tpdec) in transport component was detected at $479 \mathrm{~ms}$ and time to peak velocity (TPV) at $327 \mathrm{~ms}$. Due to this fact, very small changes are observed in the temporal occurrence of maximum grip aperture (TPA) and the onset of final grip closure relative to the overall duration of movement (see Table 1).

TABLE 1

PEAK APERTURe (MGA) AND TiMe OF OCCURRENCE (TPA)

\begin{tabular}{lcccc}
\hline & Normal & & Altered & \\
\hline & Small & Large & Small & Large \\
MGA (mm) & 51 & 96 & 66 & 106 \\
TPA (ms) & 538 & 562 & 500 & 507 \\
\hline
\end{tabular}



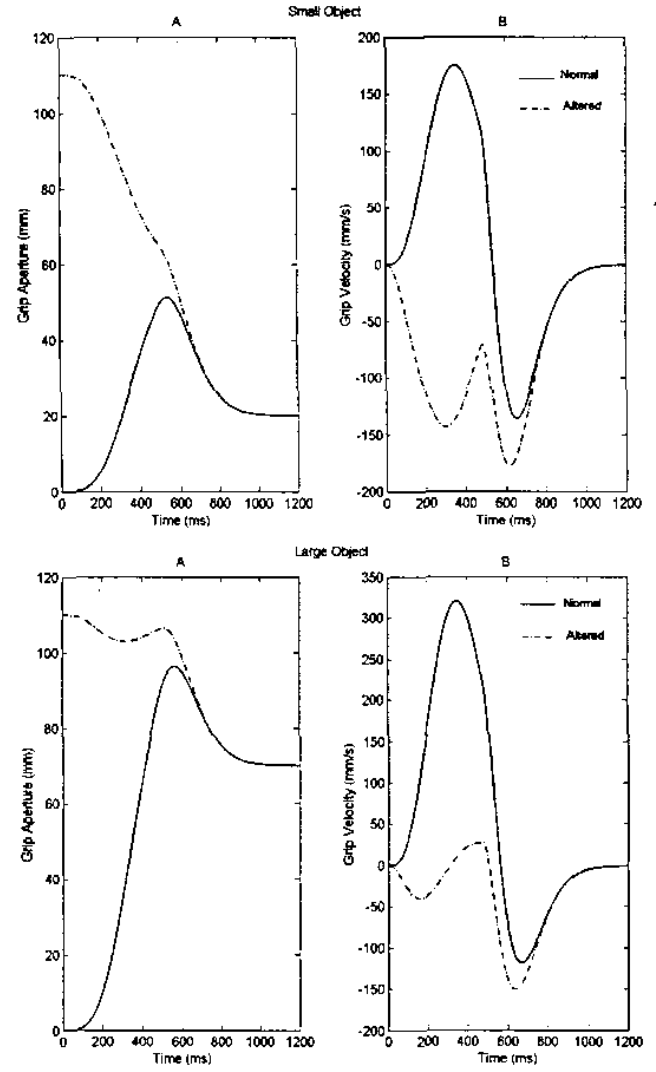

Figure 2. Normal and Altered hand initial aperture conditions. A. The change of grip aperture profile in the Altered condition (dotted line). B. Note the double peak of grip aperture velocity in the Altered condition (dotted

line) and the fitting of final peaks of grip velocity.

\subsection{Comparison with Human Data}

Simulated data match human data in many aspects. As seen in the velocity profiles of the Altered condition (see Fig 2B), the peak initial grip velocity is substantially different for each object size. The peak initial velocity of the grip for the small object is significantly faster than of the large object. As shown in Fig 3, in the Normal condition, the amplitude of the initial velocity peak for the small object is smaller than for the large object. For final velocity peak this relationship is reversed. In the Altered condition, the amplitude of the initial velocity peak for the small object is larger than for the large object. For final velocity peak this relationship is the same one. These results are in agreement with human data presented in [5]. It was also reported that in Normal condition, peak aperture exceeded $31 \mathrm{~mm}$ for the small object and $20 \mathrm{~mm}$ for the large object. In our simulations peak aperture exceeded $31 \mathrm{~mm}$ for the small object and $26 \mathrm{~mm}$ for the large object. In Altered condition, as reported in [5], the peak aperture exceeded $42 \mathrm{~mm}$ for the small object and 35 $\mathrm{mm}$ for the large one. In simulations we have obtained 46 $\mathrm{mm}$ for the small object and $36 \mathrm{~mm}$ for the large one. So, the model is able to reproduce basic kinematic features of grip formation during prehension in Normal and Altered conditions assuming the reduced set of hypothesis commented above.

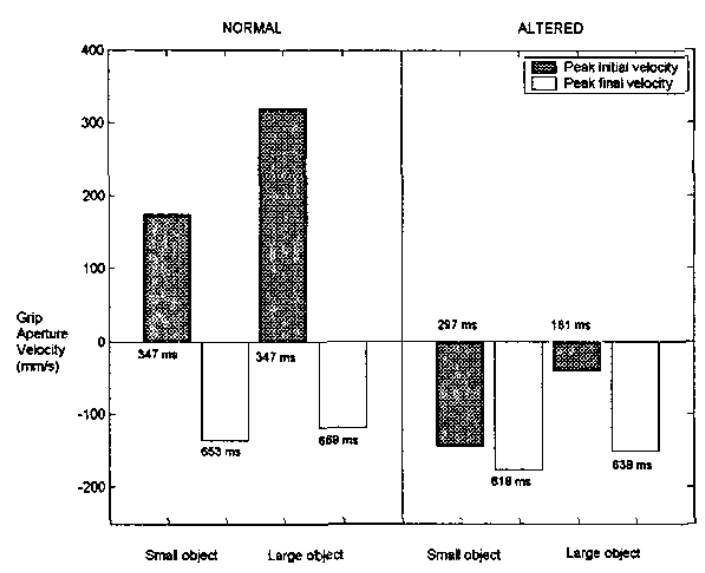

Figure 3. Amplitude of grip velocity peaks in Normal and Altered conditions. The time to grip velocity peak is given in milliseconds (ms).

\section{Conclusions}

In recent years, the interface between neuroscience and robotics is carried out by Neurorobotics research. Neurorobotics tries to investigate, model and validate neural dynamics underlying successful performance of complex tasks such as grasping and manipulative actions by humans, and transfer validated neural algorithms into the design of control algorithms acting on bio-inspired robotic platforms.

Recently, two different models [8], [9] for prehension have been presented. Both models predict an initial hand opening in the Altered initial hand aperture condition that is not present in the human data. The difference between these models is their complexity. Both models take into account the constraints of the object, and even one of them [9] also considers various anatomical and postural constraints. In our opinion both models fail in predicting the observed grip kinematics in Altered condition because both models neglect the neural dynamics underlying the prehensile behaviour.

In this paper we have presented a model that constitutes a distributed control system in which neural activity in different neural populations contribute to the specification, coordination, control and execution of complex motor tasks such as reach to grasp objects of different size. The model is based on a simple and well known neural model for trajectory formation (VITE). 
Taking into account several behavioural and neural findings, we have constructed a very simple model, able to reproduce the kinematics of reach to grasp movements observed in experiments performed by human subjects under different initial conditions. The model encapsulates the theoretical postulate that voluntary movements involve parallel volitional gated process of integrating difference vectors ( $\mathrm{V}$ vectors in the model) to update position commands [11]. It is proposed that the movement pre-shaping of hand and fingers during pre-contact phase, can be understood in terms of basic motor programs that can be spatio-temporally modulated to accommodate to different task demands [12].

The inclusion of more elaborated models of end effectors for reaching and grasping, and addition of realistic anatomical or postural constraints should increment the explanatory richness of the model and allow its integration and implementation into anthropomorphic robotic platforms for reaching and grasping.

\section{References}

[1] M. Gentilucci, U. Castiello, M.L. Corradini, M. Scarpa, C. Umiltà, G. Rizzolatti, "Influence of different types of grasping on the transport component of prehension movements," Neuropsychologia, Vol. 29, pp.361 - 378, 1991.

[2] G.E. Stelmach, U. Castiello, M. Jeannerod, "Orienting the finger opposition space during prehension movements," J. Motor Behav, Vol. 26, pp.178 - 196, 1994.

[3] M. Jeannerod, "The timing of natural prehension movements," J. Motor Behav, Vol. 16, pp.235-254, 1984

[4] M.A. Arbib, "Schemas for the temporal control of behaviour," Human Neurobioly, Vol. 4, pp.63 - 72, 1985.

[5] M. Saling, S. Mescheriakov, E. Molokanova, G.E. Stelmach, M. Berger, "Grip reorganization during wrist transport: the influence of an altered aperture," Exp Brain Res, Vol. 108, 493-500, 1996.

[6] D. Timman, G.E Stelmach, J.R. Bloedel, "Grasping component alterations and limb transport," Exp Brain Res, Vol. 108, pp.486-492, 1996.

[7] S.A. Wallace, D.L. Weeks, J.A.S. Kelso, "Temporal constraints in reaching and grasping behavior," Hum Mov Science, Vol. 9, pp.69-93, 1990.

[8] J.B.J Smeets, E. Brenner, "A new view on grasping," Motor Control, Vol. 3, pp.237-271, 1999.
[9] R.G.J. Meulenbroek, D.A. Rosenbaum, C. Jansen, J. Vaughan, S. Vogt, "Multijoint grasping movements: Simulated and observed effects of object location, object size and initial aperture," Exp Brain Res, Vol. 138 (2), pp.219-234, 2001.

[10] J.B.J Smeets, E. Brenner, "Does a complex model help to understand grasping?," Exp Brain Res, Vol. 144, pp. 132-135, 2002.

[11] D. Bullock, S. Grossberg, "Neural dynamics of planned arm movements: emergent invariants and speedaccuracy properties during trajectory formation," Psychol Rev, Vol. 95, pp.49-90, 1988.

[12] J.L. Contreras-Vidal, A. Ulioa-Pérez, J. LópezCoronado, "Neural dynamics of hand preshaping during prehension," IEEE Conference on Systems, Man, and Cybernetics, Tucson, AZ, vol 5, pp. 3019-3024, 2001.

[13] M. Santello, J.F. Soechting, "Gradual molding of the hand to object contours," J. Neurophysiol, Vol. 79, pp.1307-1320, 1998.

[14] J.L. Contreras-Vidal, G.E. Stelmach, "A neural model of basal ganglia-thalamocortical relations in normal and Parkinsonian movement," Biol Cyb, Vol. 73, pp. 467$476,1995$.

[15] J. Molina Vilaplana, J. Feliu, J. López Coronado, "A neural model of spatio temporal coordination in prehension," Lec Not in Comp Sci, Vol. 2415, pp.9-14. 2002.

[16] Q.G. Fu, J.I. Suarez, T.J Ebner, "Neuronal specification of direction and distance during reaching movements in the superior precentral area and primary motor cortex of monkeys," J. Neurophysiol, Vol. 70, pp.2096-2126, 1993.

[17] M. Simoneau, J. Paillard, C. Bard, N. Teasdale, O. Martin, M Fleury, Y. Lamarre, "Role of the feedforward command and reafferent information in the coordination of a passing prehension task," Exp Brain Res, Vol. 128, pp. $236-242,1999$.

[18] P. Cordo, M. Schieppati, L. Bevan, L.G. Cariton, M.J. Carlton, "Central and peripheral coordination in movement sequences," Psychol Res, Vol. 55, pp.124-130, 1993.

[19] E.B. Motgomery, D.S. Gorman, J. Nuessen, "Motor initiation versus execution in normal and Parkinson's disease subjects," Neurology, Vol. 41, pp.1469-1475, 1991. 